Artículo científico

Volumen 32(1):1-18. Enero-abril, 2021

e-ISSN 2215-3608, doi:10.15517/am.v32i1.41928

https://www.revistas.ucr.ac.cr/index.php/agromeso

\title{
Frecuencia de fotogramas óptima para evaluar la cinética espermática de verracos con un sistema CASA-Mot ${ }^{1}$
}

\section{Optimal frame rate to evaluate boar sperm kinematic with a CASA-Mot system}

\author{
Vinicio Barquero' ${ }^{2}$ Luis Víquez ${ }^{2}$, Josué Calderón-Calderón ${ }^{2}$,Anthony Valverde ${ }^{2,3}$
}

1 Recepción: 27 de agosto, 2020. Aceptación: 16 de septiembre, 2020. Este trabajo formó parte del proyecto de investigación "CASA-Mot technology" desarrollado en el Departamento de Biología Celular y Antropología Física de la Universidad de Valencia, España.

2 Instituto Tecnológico de Costa Rica (ITCR), Escuela de Agronomía, Centro de Investigación y Desarrollo en Agricultura Sostenible del Trópico Húmedo, Campus Tecnológico Local San Carlos. Apdo. Postal 223-21002 Alajuela, Costa Rica. vinicio1196@gmail.com (https:// orcid.org/0000-0003-0042-6178); luisga.viquez@gmail.com (https://orcid.org/0000-0002-8115-441X); ccjosuedavid@gmail.com (https:// orcid.org/0000-0001-6860-217X), anvalverde@itcr.ac.cr (autor para la correspondencia, https://orcid.org/0000-0002-3191-6965).

3 Universidad de Valencia, Departamento de Biología Celular, Biología Funcional y Antropología Física, Campus Burjasot, Valencia, España.

\section{Resumen}

Introducción. La movilidad es el indicador de calidad seminal más utilizado, por tanto, es necesario determinar una adecuada frecuencia de fotogramas (frame rate, FR) para estandarizar los análisis cinéticos con los equipos de análisis computadorizado de semen (CASA-Mot) y disminuir la variabilidad entre laboratorios, lo que optimizará el número de dosis seminales y su calidad para la industria porcina basada en inseminación artificial (IA). Objetivo. Determinar la frecuencia óptima de fotogramas sobre la cinética espermática de verraco mediante un sistema comercial CASA-Mot y analizar la distribución de subpoblaciones espermáticas con el FR óptimo. Materiales y métodos. Se utilizaron veinte dosis seminales de diez verracos Pietrain (Sus scrofa domestica). El periodo experimental fue entre febrero y julio del año 2017. Se utilizó el sistema CASA-Mot (ISAS ${ }^{\circledR}$ v1) con cámaras de recuento ISAS ${ }^{\circledR}$ D4C20 precalentadas a $37{ }^{\circ} \mathrm{C}$. El tiempo de captura fue de dos segundos. Resultados. La frecuencia de fotogramas afectó los parámetros de cinética espermática, exceptuando la velocidad rectilínea (VSL), debido a que no representó un comportamiento dependiente del FR. Las demás variables presentaron alteraciones significativas al cambiar su FR, y la velocidad curvilínea (VCL) y velocidad promedio (VAP) fueron las variables más sensibles al cambio. Se obtuvieron tres componentes principales (PC) definidos por patrones de movilidad, progresividad y oscilación, en donde el PC1 (velocidad-ondulación) fue el más influyente, debido a que representó el 51,34 \% y 55,08 \% de la varianza total explicada para 25 y 200 fps (fotogramas por segundo), respectivamente. Conclusión. La frecuencia óptima de fotogramas para analizar variables de cinética espermática en verraco con un sistema CASA-Mot fue de $200 \mathrm{fps}$. Una tasa de fotogramas inferior provoca errores en la estimación de la trayectoria de los espermatozoides. La distribución de subpoblaciones espermáticas varía según la tasa de fotogramas utilizada. Es necesario establecer un protocolo de análisis para homogenizar los resultados.

Palabras clave: espermatozoo, fotogramas por segundo, subpoblaciones espermáticas, cerdo, reproducción porcina. 


\begin{abstract}
Introduction. Motility is the most used seminal quality indicator, therefore, it is necessary to determine an adequate frame rate (FR) to standardize kinetic analysis with computerized semen analysis equipment (CASA-Mot) and reduce variability between laboratories, which will improve the number of seminal doses and their quality for the swine industry based on artificial insemination (AI). Objective. To determine the optimal frame rate frequency on boar sperm kinetics using a commercial CASA-Mot system and to analyze the distribution of sperm subpopulations with the optimal FR. Materials and methods. Twenty seminal doses of ten Pietrain boars (Sus scrofa domestica) were used. The experimental period was between February and July 2017. The CASA-Mot system (ISAS ${ }^{\oplus} \mathrm{V} 1$ ) was used with ISAS ${ }^{\oplus} \mathrm{D} 4 \mathrm{C} 20$ counting chambers preheated to $37^{\circ} \mathrm{C}$. The video capture time was two seconds. Results. The frame rate affected the sperm kinetic parameters, except for the rectilinear velocity (VSL), because it did not represent a FR-dependent behavior. The other variables presented significant alterations when changing their FR, and curvilinear velocity (VCL) and average path velocity (VAP) were the most sensitive values to the change. Three principal components (PC) defined by patterns of motility, progressiveness and oscillation were obtained, where the PC1 (velocity-undulation) was the most influential since it represented $51.34 \%$ and $55.08 \%$ of the total explained variance for 25 and $200 \mathrm{fps}$ (frames per second), respectively. Conclusion. The optimal frame rate to analyze sperm kinematics variables in boar semen with a CASA-Mot system was $200 \mathrm{fps}$. A lower frame rate causes errors in the sperm trajectory estimation. The sperm subpopulation distribution changes according to the frame rate used. It is necessary to establish an analysis protocol to homogenize the results.
\end{abstract}

Keywords: spermatozoa, frames per second, sperm subpopulations, pig, swine reproduction.

\title{
Introducción
}

La evaluación de la calidad seminal permite estimar el potencial reproductivo del macho mediante una muestra del eyaculado (Parrilla et al., 2019). Con el surgimiento de los sistemas computarizados de análisis seminal (Computer Assisted Semen Analysis, CASA), se ha logrado una mayor estandarización de las evaluaciones, debido a que los desarrolladores de sistemas CASA han establecido configuraciones específicas para algunas especies de interés zootécnico (Amann \& Waberski, 2014). No obstante, estos deben ser validados para cada especie.

El análisis de la movilidad espermática se basa en parámetros de cinética (Lu et al., 2014; Valverde \& MadrigalValverde, 2018). La funcionalidad básica de esta medición se debe a la captura de fotogramas sucesivos para la construcción de la trayectoria del espermatozoide (Katz \& Dott, 1975; Mortimer, 2000; Valverde \& MadrigalValverde, 2018). Los sistemas CASA-Mot han demostrado gran exactitud en la evaluación de la movilidad y cinética espermática en comparación con los métodos subjetivos de valoración seminal por técnicos de laboratorio (Amann \& Waberski, 2014), sin embargo, proveen gran cantidad de información (Valverde et al., 2019c) que debe ser analizada por medio de estadística multivariada (Spencer, 2013) para condensar toda la información obtenida y reducir la dimensionalidad de las variables cinéticas.

A pesar de que la evaluación con sistemas CASA es más confiable en comparación con la evaluación manual, los resultados pueden ser influenciados por diversos factores como la configuración del software (Amann \& Katz, 2004), número de campos analizados (Valverde \& Madrigal-Valverde, 2019), tiempo transcurrido (Valverde et al., 2019c) y cámara de recuento espermático (Soler et al., 2012; Bompart et al., 2019; Caldeira et al., 2019). Además, en el mercado se cuenta con variedad de equipos que pueden generar resultados disímiles al compararse, lo que genera controversias (Bompart et al., 2018; Morris et al., 1996; Rijsselaere et al., 2004; Valverde et al., 2020).

Como alternativa para reducir la influencia de los factores anteriormente mencionados y las diferencias entre sistemas CASA-Mot utilizados, se deben investigar las interacciones con los factores para lograr establecer una 
configuración de trabajo adecuada para cada especie a analizar. Algunos autores han identificado la necesidad de definir la altura de cámara de recuento con la cual es recomendable el análisis, gracias a ello, se ha demostrado que para la especie porcina es más favorable utilizar cámaras de $20 \mu \mathrm{m}$ de profundidad, debido a que esta amplitud proporciona el espacio óptimo para promover el movimiento de los espermatozoides (Castellini et al., 2011; Valverde \& Madrigal-Valverde, 2019).

El análisis de semen en granjas que se dedican a la venta de dosis seminales o a la inseminación artificial (IA) permite optimizar el número de dosis por macho (Valverde \& Madrigal-Valverde, 2019) y reducir los costos de manutención de los animales (Kime et al., 2001; Verstegen et al., 2002; Knox, 2016; Lopez-Rodriguez et al., 2017; Fair \& Romero-Aguirregomezcorta, 2019). Los métodos objetivos de análisis de semen o sistemas CASA, en donde el CASA-Mot es el más utilizado, proporcionan información sobre la movilidad de una muestra y variables de cinética espermática para caracterizar los eyaculados (Amann \& Waberski, 2014). No obstante, los resultados obtenidos de estas evaluaciones se ven influenciados por diversos factores como lo son la concentración (Saravia et al., 2007), tipo y profundidad de la cámara de recuento espermático (Bompart et al., 2018; Saravia et al., 2007; Valverde \& Madrigal-Valverde, 2019), el campo de observación (Nöthling \& dos Santos, 2012), el tiempo de captura (Valverde et al., 2019c) y la frecuencia de fotogramas (Wilson-Leedy \& Ingermann, 2007; Bompart et al., 2019; Valverde et al., 2019a).

Como medida para contrarrestar la heterogeneidad de los resultados, se debe establecer un protocolo a seguir con las condiciones de trabajo adecuadas para la especie porcina (Sus scrofa domestica) (Amann \& Waberski, 2014). Algunas condiciones han sido previamente estudiadas y favorecen el establecimiento de estos protocolos para obtener datos menos disímiles, como es el caso de la altura de la cámara de recuento espermático que se ha establecido que a $20 \mu \mathrm{m}$ favorece el movimiento de las células respecto de cámaras con profundidades menores (Valverde \& Madrigal-Valverde, 2019). Otros factores como la frecuencia de fotogramas se ha estudiado en espermatozoides de verraco y se ha determinado que para analizar la movilidad de estos, se pueden utilizar utilizar $50 \mathrm{fps}$, debido a que no representa diferencias significativas con respecto al incremento de la frecuencia de fotogramas (FR) (Valverde et al., 2019a). No obstante, los parámetros de cinética requieren cámaras de mayor resolución en donde se pueda utilizar un FR mayor para lograr más precisión y exactitud en la estimación de las trayectorias reales de la célula, lo que conlleva un alto grado de confiabilidad (Mortimer \& Swan, 1999; Valverde et al., 2019a). Para la predicción de la trayectoria real de los espermatozoides, se debe realizar la evaluación de la cinética bajo condiciones de trabajo estandarizadas (Bompart et al., 2018; Gallagher et al., 2019). Por ejemplo, al construir las trayectorias celulares es necesario partir de un archivo de video en donde se obtengan la movilidad de los espermatozoides.

Hay que definir criterios óptimos en relación con el tiempo de captura de video para estandarizar obtenga protocolos de análisis. Respecto de la movilidad total de la muestra se puede utilizar un tiempo de captura de 0,5 $\mathrm{s}$, mientras que si se requieren conocer las variables de cinética es preferible utilizar $2 \mathrm{~s}$ de captura (Valverde et al., 2019c). Si la memoria de cálculo no es un factor limitante, es preferible capturar el mayor tiempo posible con un elevado número de fotogramas por segundo, y se ha estimado que una captura de video durante $2 \mathrm{~s}$ a $200 \mathrm{fps}$, según la base de estos resultados, es adecuado o a 225 fps (Valverde et al., 2019a). Para evaluar comercialmente la movilidad se podrá tomar como referencia el tiempo indicado de $0,5 \mathrm{~s}$ de captura a 50 fps (Valverde et al., 2019c).

En cuanto a la frecuencia de fotogramas (FR), se ha determinado que la mayoría de las variables de cinética se han visto incrementadas al aumentar los fotogramas por segundo (fps) (Valverde et al., 2019a). Para obtener resultados más confiables, es necesario que los análisis se realicen en condiciones que generen la mayor precisión y exactitud, debido a esto es requerida una adecuada elección del número de fotogramas por segundo para proveer confianza al investigador (Bompart et al., 2018; Valverde et al., 2019a).

Cuando se estudia la distribución de agrupaciones celulares dentro de un eyaculado o subpoblaciones espermáticas, los factores externos a estos pueden condicionar la cantidad de conjuntos celulares o clusters que 
se encuentran en una muestra. Los factores externos deben ser conocidos para determinar con mayor certeza la distribución real de subpoblaciones celulares dentro del eyaculado (Ibanescu et al., 2020). Desde hace tiempo se reconoce que el eyaculado no es uniforme (Martínez-Pastor et al., 2011). Existen conjuntos celulares con diferentes patrones de movimiento y cinemáticos que están interactuando para asegurar el éxito de la fecundación (Yániz et al., 2017; Peña et al., 2018). El estudio de las subpoblaciones espermáticas ha sido posible por la gran cantidad de datos que aportan los sistemas CASA al analizar miles de espermatozoides en un corto periodo. Como resultado del análisis espermático por sistemas CASA, se ha determinado que las variables cinéticas de los espermatozoides no se distribuyen según una función de distribución normal, sino que son disímiles y se agrupan en diferentes subpoblaciones definidas por sus características de movilidad y cinética (Amann \& Hammerstedt, 1993; Hirai et al., 2001; Soler et al., 2014; Gallego et al., 2015; Valverde et al., 2016; Vásquez et al., 2016; Yániz et al., 2016; Soler et al., 2017; Peña et al., 2018; Valverde et al., 2018; van der Horst et al., 2018; Yániz et al., 2018; Caldeira et al., 2019; García-Molina et al., 2019).

En diversas especies se ha determinado que existen subpoblaciones espermáticas caracterizadas por sus variables cinéticas (Amann \& Hammerstedt, 1993; Hirai et al., 2001; Thurston et al., 2001; Flores et al., 2008; Ramió et al., 2008; Soler et al., 2014; Gallego et al., 2015; Valverde et al., 2016; Vásquez et al., 2016; Yániz et al., 2016; Soler et al., 2017; Valverde et al., 2018; Yániz et al., 2018; Caldeira et al., 2019; Valverde et al., 2019b; Víquez et al., 2020). En la especie porcina, trabajos previos han descrito subpoblaciones espermáticas en función de la frecuencia de fotogramas (Valverde et al., 2019a), sin embargo, es necesario determinar la consistencia de las agrupaciones celulares en los eyaculados para determinar si existe un patrón característico en la distribución de las subpoblaciones espermáticas.

El objetivo del presente estudio fue determinar la frecuencia óptima de fotogramas sobre la cinética espermática de verraco mediante un sistema comercial CASA-Mot y analizar la distribución de subpoblaciones espermáticas dentro de los eyaculados con la FR óptima.

\section{Materiales y métodos}

\section{Descripción y localización del experimento}

El estudio se llevó acabo en el Laboratorio de Andrología de Proiser R+D, ubicado en el parque científico de la Universidad de Valencia, Campus de Burjassot, Valencia, España. Los análisis de cinética espermática se desarrollaron de febrero a julio del año 2017. Las muestras de semen de verraco se mantuvieron en condiciones controladas de temperatura y almacenamiento en el laboratorio, con el propósito de no introducir fuentes de variación en la movilidad seminal.

\section{Animales}

Se utilizaron veinte dosis seminales, obtenidas de diez verracos (Sus scrofa domestica) donadores de la raza Pietrain, cuyas edades variaron entre dos y tres años, lo que permitió categorizarlos como verracos adultos. Los animales fueron alojados en corrales con condiciones climatizadas en la granja núcleo comercial de Semen Cardona, S.L. (Cardona, Barcelona, España). Fueron alimentados con una dieta comercial para verracos reproductores ajustada $\left(2,3 \mathrm{~kg} \mathrm{~d}^{-1}\right)$ y provistos de agua ad libitum. 


\section{Recolección de muestras seminales y procesamiento}

Se recolectaron muestras de semen en fresco diluidas 1:1 (vol:vol), en un diluyente comercial (BTS, Minitüb, Alemania) para preservación de semen porcino durante tres días. El mismo técnico realizaba las extracciones seminales a los verracos. Antes de cada extracción seminal, los verracos se trasladaron a un corral de monta donde se procedía a extraer el semen mediante la técnica del doble guante. La concentración espermática se determinó mediante espectrofotometría. Se realizó el cómputo del número total de espermatozoides, pre multiplicando la concentración por el volumen del eyaculado. Las muestras de semen que se utilizaron superaron el umbral mínimo de calidad seminal, es decir, presentaron un mínimo de $85 \%$ de morfología normal, $85 \%$ de movilidad total y una concentración de espermatozoides superior a $225 \times 10^{6}$ espermatozoides $\mathrm{ml}^{-1}$. El transporte de las muestras al laboratorio se realizó en condiciones de refrigeración $\left(17^{\circ} \mathrm{C}\right)$, de la misma manera en que se realiza la distribución comercial. Al llegar al laboratorio las muestras se agitaron y, posteriormente se tomó una alícuota de $1 \mathrm{ml}$, se colocaron en microtubos para centrífuga de $1 \mathrm{ml}$ y se mantuvieron a $37^{\circ} \mathrm{C}$ durante 30 min antes de su análisis espermático.

\section{Evaluación del semen con el sistema CASA-Mot}

El análisis de la cinética espermática se realizó mediante la utilización de cámaras de recuento desechables ISAS ${ }^{\oplus} 4 \mathrm{C} 20$ (Proiser R + D, S.L., Paterna, España), de $20 \mu \mathrm{m}$ de altura, las cuales se precalentaron a $37{ }^{\circ} \mathrm{C}$. Después de la homogenización de las muestras, se distribuyó por capilaridad un volumen de semen de $3 \mu \mathrm{l}$ a lo largo de la cámara de recuento. Todos los análisis se realizaron con el sistema CASA-Mot ISAS ${ }^{\oplus} \mathrm{v} 1$ (Integrated Semen Analysis System, Proiser R + D, Paterna, España). Se utilizó una cámara de video de alta velocidad (500 fps, M03-CM, Proiser R + D) para obtener las imágenes. La frecuencia de fotogramas se definió con una frecuencia de captura variable de 25, 50, 100, 150, 200 y 250 fotogramas por segundo (frame per second, fps), el tiempo de captura fue de $2 \mathrm{~s}$; la resolución final de los videos fue de 648 x 488 píxeles x 8 bits, con una escala de gris de 256. La resolución de las imágenes fue de $0,70 \mu \mathrm{m}$ por pixel en los ejes horizontal y vertical. La instalación de detección de colas del sistema se activó para evitar la detección de partículas no espermáticas, con un área de partículas entre 10 y $80 \mu \mathrm{m}^{2}$ y un valor de conectividad de $11 \mu \mathrm{m}$. La cámara se conectó a un microscopio UB203 (UOP / Proiser R + D) con un ocular de $1 \mathrm{X}$, un objetivo de contraste de fase negativo 10X $(\mathrm{AN} 0,25)$ y una placa calefactora integrada mantenida a $37,0 \pm 0,5^{\circ} \mathrm{C}$. La configuración del equipo utilizada fue la recomendada por el fabricante según la especie porcina. Para evaluar las muestras se utilizó una computadora con un procesador Intel ${ }^{\circledR}$ (Intel Corporation, California, Estados Unidos) Core i3 de 3,30 GHz, 4 GB de memoria RAM, disco duro de 452 GB y sistema de 64 bits. Los errores de reconocimiento de las trayectorias las revisó el mismo técnico y los eliminó cuando fue necesario, para evitar la introducción de distorsiones en los resultados finales.

\section{Análisis de variables de cinética espermática}

De cada muestra analizada se realizaron dos repeticiones y se tomaron siete campos a lo largo de la cámara de recuento, para alcanzar un mínimo de 600 espermatozoides por muestra. Las variables cinéticas consideradas en el estudio fueron: la velocidad rectilínea (VSL, $\mu \mathrm{m} \mathrm{s}^{-1}$ ), correspondiente a la línea recta desde el principio hasta el final de la trayectoria; la velocidad curvilínea (VCL, $\mu \mathrm{m} \mathrm{s}^{-1}$ ), medida sobre la trayectoria real de punto a punto seguida por el espermatozoide; la velocidad de trayectoria promedio (VAP, $\mu \mathrm{m} \mathrm{s}^{-1}$ ), calculada como una interpolación entre los puntos correspondientes a la trayectoria de la VCL; la amplitud de desplazamiento lateral de la cabeza (ALH, $\mu \mathrm{m}$ ), expresada como la altura máxima (o media) de la amplitud del movimiento oscilatorio de la trayectoria curvilínea; y la frecuencia de entrecruzamiento $(\mathrm{BCF}, \mathrm{Hz})$, expresada como el número de veces que la trayectoria curvilínea cruza la lineal. La configuración del software se ajustó al análisis de espermatozoides de 
verraco: de 10 a $80 \mu \mathrm{m}^{2}$, para el área de la cabeza, y 11 para conectividad. Además, se estimaron tres relaciones de progresión expresadas como porcentajes, a partir de las mediciones de velocidad descritas anteriormente: linealidad de la progresión directa $(\mathrm{LIN}=\mathrm{VSL} / \mathrm{VCL} * 100)$, rectitud $(\mathrm{STR}=\mathrm{VSL} / \mathrm{VAP} * 100)$ e índice de oscilación (WOB $=\mathrm{VAP} / \mathrm{VCL} * 100)$.

\section{Análisis estadístico}

Los datos obtenidos del análisis de todas las variables espermáticas se evaluaron primero para determinar la normalidad y la homocedasticidad mediante las pruebas de Shapiro-Wilks y Levene. Se utilizó, además, papel probabilístico normal para evaluar la distribución normal. Se determinó el efecto de la frecuencia de fotogramas sobre las variables cinéticas a través de un ANOVA de una vía. En los casos pertinentes, se aplicó la prueba de Bonferroni para el análisis a posteriori de las variaciones entre el número de fotogramas capturados por segundo.

Para los análisis de regresión, los efectos de la FR se evaluaron con un modelo exponencial, de la forma $y=$ $\beta * \alpha \exp (-\beta / x)$, donde $y$ es VCL, $x$ es FR, $\alpha$ es el nivel asintótico, $\beta$ es la tasa de aumento a la asíntota, y $\exp$ es la base de los logaritmos naturales. La importancia biológica de la ecuación es que los valores asintóticos para $\alpha$ representan el máximo alcanzable cuando el FR está por encima del valor umbral. El valor umbral se calculó como la FR necesaria para obtener el $95 \%$ del valor máximo. La tasa de aproximación a la asíntota representa la dependencia de la curva con la FR; es decir, un valor relativamente mayor de $\beta$ indica un mayor aumento de VCL a medida que aumenta la FR y viceversa.

Se realizaron procedimientos multivariados para identificar subpoblaciones de esperma del conjunto de datos de cinética espermática a 25 y 200 fps, considerado como el óptimo mínimo de la FR. Todos los valores para las variables cinemáticas se estandarizaron para evitar cualquier efecto de escala. El primer proceso consistió en realizar un análisis de componentes principales (PCA) de estos datos para obtener un pequeño número de combinaciones lineales que aún conservaban la mayor cantidad de información posible de las variables originales. El número de componentes principales (PC) utilizados en el siguiente proceso del análisis, se determinó a partir del criterio de Kaiser, es decir, seleccionando solo aquellos con un valor propio (varianza extraída de cada PC)> 1. Además, se realizaron la prueba de esfericidad de Bartlett y el KMO (Kaiser-Meyer-Olkin) (Spencer, 2013). Como método de rotación, se utilizó el método varimax con la normalización de Kaiser (Kaiser, 1958). El segundo proceso se realizó para realizar un análisis no jerárquico con el modelo de k-means que utiliza distancias euclidianas de las variables cuantitativas después de la estandarización de estos datos, por lo que los centros de agrupación fueron las medias de las observaciones asignadas a cada agrupación (Kaufman \& Rousseuw, 1991).

El análisis multivariado de grupos de k-means se realizó para clasificar los espermatozoides en un número reducido de subpoblaciones (SP, grupos), de acuerdo con sus variables cinemáticas estructuradas en vectores de componentes principales. En el proceso final, para determinar el número óptimo de grupos, los centroides finales se agruparon jerárquicamente utilizando el método Ward (Murtagh \& Legendre, 2014). Por lo tanto, el procedimiento de agrupamiento permitió la identificación de subpoblaciones espermáticas, porque cada grupo contribuyó a un grupo final formado por los espermatozoides unidos a los centroides. Los procedimientos de prueba ANOVA se aplicaron para evaluar las diferencias estadísticas en la distribución de observaciones (espermatozoides individuales) dentro de las variables cinéticas que definen las diferentes subpoblaciones de esperma (es decir, los centros de agrupación).

Las diferencias entre medias se analizaron mediante la prueba de Bonferroni. Los resultados se presentaron como medias \pm error estándar de la media (EEM). La significancia estadística se consideró en $\mathrm{p} \leq 0,05$. Todos los datos se analizaron con el paquete IBM SPSS, versión 23.0 para Windows (SPSS Inc., Chicago, Il, EE. UU.). 


\section{Resultados}

\section{Distribución de la cinética espermática según frecuencia de fotogramas}

La velocidad rectilínea (VSL) fue la variable que menos se vio afectada por los incrementos en la frecuencia de fotogramas, sin embargo, la variación porcentual entre 150 y $200 \mathrm{fps}$ fue de 8,30 \%. La velocidad curvilínea (VCL) se incrementó ( $\mathrm{p}<0,05)$ al aumentar la FR, pasando de $48,17 \pm 0,79 \mu \mathrm{m} \mathrm{s}^{-1}$ con $25 \mathrm{fps}$, a $157,42 \pm 0,90 \mu \mathrm{m} \mathrm{s}^{-1}$ con $250 \mathrm{fps}$, lo que significó un incremento porcentual de 226,80 \%. Por su parte, la velocidad promedio (VAP) también se incrementó ( $\mathrm{p}<0,05)$ al aumentar la FR, pasando de $40,41 \pm 0,47 \mu \mathrm{m} \mathrm{s}^{-1}$ con $25 \mathrm{fps}$, a $82,32 \pm 0,54 \mu \mathrm{m} \mathrm{s}^{-1}$ con $250 \mathrm{fps}$, lo que representó un incremento porcentual de 103,71\%. Los índices de linealidad (LIN) y rectitud (STR) disminuyeron $(\mathrm{p}<0,05)$ conforme se incrementó el número de fotogramas por segundo, no obstante, el índice de oscilación (WOB) presentó diferencias $(\mathrm{p}<0,05)$ cuando las frecuencias de fotogramas fueron menores hasta $100 \mathrm{fps}$, y por encima de este nivel los resultados no mostraron una variación biológica relevante (Figura 1). La amplitud del desplazamiento lateral de la cabeza $(\mathrm{ALH})$ presentó diferencias $(\mathrm{p}<0,05)$ relevantes entre 25 fps
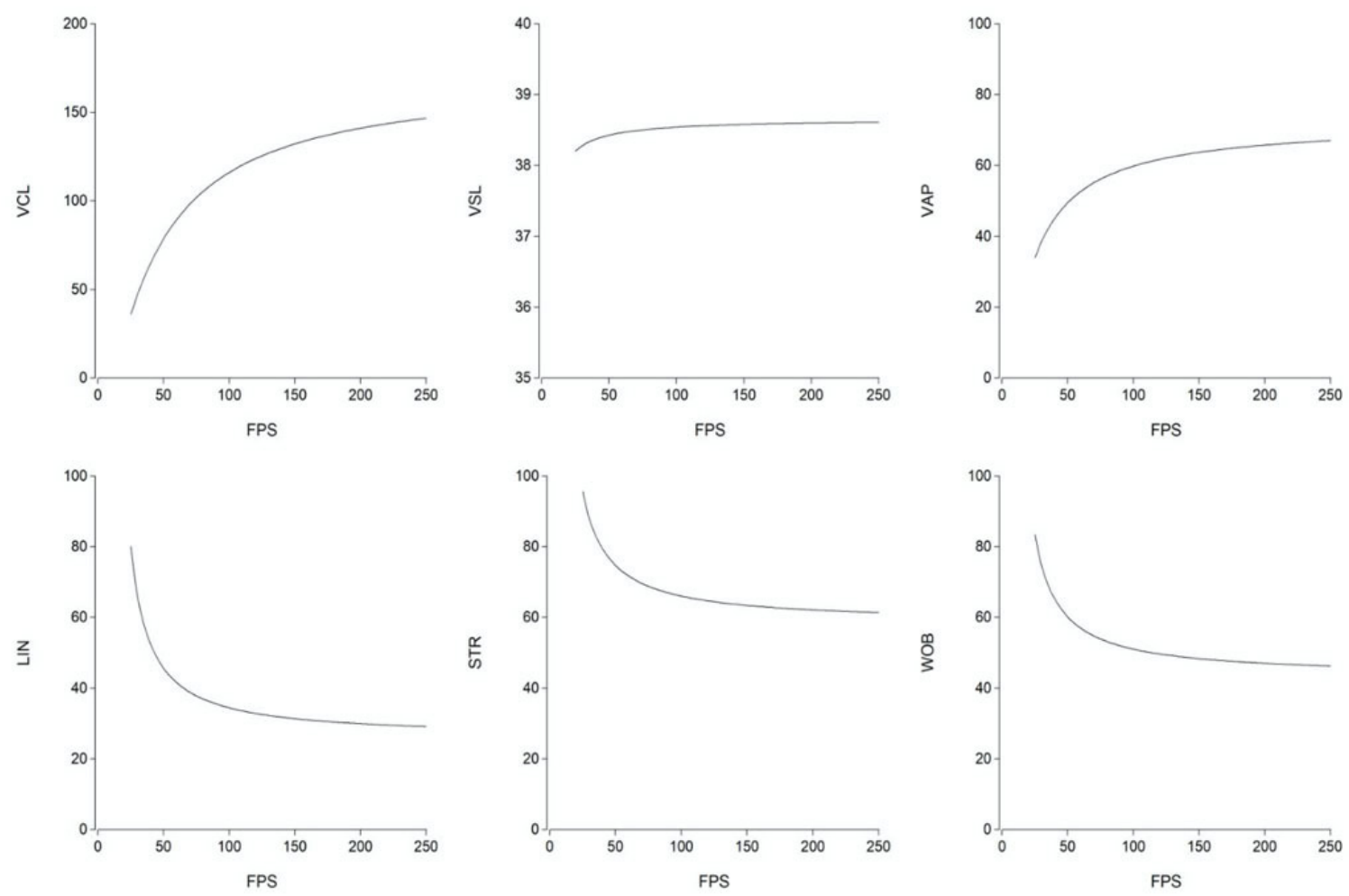

Figura 1. Efecto de la frecuencia de fotogramas sobre los valores de velocidad curvilínea (VCL, $\mu \mathrm{m} \mathrm{s}^{-1}$ ), velocidad rectilínea (VSL, $\mu \mathrm{m} \mathrm{s}^{-1}$ ), velocidad promedio (VAP, $\mu \mathrm{m} \mathrm{s}^{-1}$ ), índice de linealidad (LIN, \%), índice de rectitud (STR, \%) e índice de oscilación (WOB, \%) del semen de verracos Pietrain (Sus scrofa domestica). Valencia, España. 2017.

Figure 1. Frame rate effect on the values of curvilinear velocity (VCL, $\mu \mathrm{m} \mathrm{s}^{-1}$ ), rectilinear velocity (VSL, $\mu \mathrm{m} \mathrm{s}^{-1}$ ), average path velocity (VAP, $\mu \mathrm{m} \mathrm{s}^{-1}$ ), linearity index (LIN, \%), straightness index (STR, \%), and wobble index (WOB, \%) on Pietrain boars (Sus scrofa domestica) sperm. Valencia, Spain. 2017. 
$(1,52 \pm 0,01 \mu \mathrm{m}), 50 \mathrm{fps}(1,57 \pm 0,01 \mu \mathrm{m})$ y $200 \mathrm{fps}(1,16 \pm 0,01 \mu \mathrm{m})$, estas diferencias a valores intermedios no fueron evidentes (100-200 fps). La frecuencia de entrecruzamiento (BCF) aumentó ( $\mathrm{p}<0,05)$ conforme se incrementó la frecuencia de fotogramas, hasta $100 \mathrm{fps}$. Las variaciones encontradas por encima de $100 \mathrm{fps}$, aunque significativas, no indicaron alguna tendencia biológica relevante (Cuadro 1).

Cuadro 1. Efecto de la frecuencia de fotogramas (FR) sobre las mediciones de movilidad y cinética espermática de verracos raza Pietrain (Sus scrofa domestica), (medias \pm EEM, $\mathrm{n}=16$ 562, variación porcentual con respecto a 25 fotogramas por segundo en paréntesis). Valencia, España. 2017.

Table 1. Effect of frame rate (FR) frequency on Pietrain boar (Sus scrofa domestica) sperm motility and kinematic measurements (means \pm SEM, $n=16562$, percentage variation with respect to $25 \mathrm{fps}$ in brackets). Valencia, Spain. 2017.

\begin{tabular}{|c|c|c|c|c|c|c|}
\hline \multirow[b]{2}{*}{ Variable } & \multicolumn{6}{|c|}{ FR (fps) } \\
\hline & 25 & 50 & 100 & 150 & 200 & 250 \\
\hline VCL & $48,17 \pm 0,79^{\mathrm{a}}$ & $70,03 \pm 0,77^{\mathrm{b}}(45,4)$ & $\begin{array}{c}113,13 \pm 0,78^{\mathrm{c}} \\
(134,9)\end{array}$ & $\begin{array}{c}124,05 \pm 0,83^{\mathrm{d}} \\
(157,5)\end{array}$ & $\begin{array}{c}144,76 \pm 0,87^{\mathrm{e}} \\
(200,5)\end{array}$ & $\begin{array}{c}157,42 \pm 0,90^{\mathrm{f}} \\
(226,8)\end{array}$ \\
\hline VSL & $38,21 \pm 0,38^{\mathrm{ab}}$ & $38,49 \pm 0,37^{\mathrm{a}}(0,7)$ & $39,08 \pm 0,38^{\mathrm{a}}(2,3)$ & $36,62 \pm 0,40^{\mathrm{b}}(-4,2)$ & $39,05 \pm 0,42^{\mathrm{a}}(2,2)$ & $39,66 \pm 0,44^{\mathrm{a}}(3,8)$ \\
\hline VAP & $40,41 \pm 0,47^{\mathrm{a}}$ & $44,42 \pm 0,46^{\mathrm{b}}(9,9)$ & $57,72 \pm 0,47^{\mathrm{c}}(42,8)$ & $56,37 \pm 0,50^{\mathrm{d}}(39,5)$ & $68,18 \pm 0,52^{\mathrm{e}}(68,7)$ & $82,32 \pm 0,54^{\mathrm{f}}(103,7)$ \\
\hline LIN & $77,71 \pm 0,26^{\mathrm{a}}$ & $55,03 \pm 0,26^{\mathrm{b}}(-29,2)$ & $34,11 \pm 0,26^{c}(-56,1)$ & $29,07 \pm 0,27^{\mathrm{d}}(-62,6)$ & $26,55 \pm 0,29^{\mathrm{e}}(-65,8)$ & $24,79 \pm 0,30^{\mathrm{f}}(-68,1)$ \\
\hline STR & $91,42 \pm 0,31^{\mathrm{a}}$ & $84,07 \pm 0,31^{\mathrm{b}}(-8,0)$ & $72,65 \pm 0,31^{\mathrm{c}}(-20,5)$ & $63,98 \pm 0,33^{\mathrm{d}}(-30,0)$ & $58,06 \pm 0,35^{\mathrm{e}}(-36,5)$ & $48,48 \pm 0,36^{\mathrm{f}}(-47,0)$ \\
\hline WOB & $82,87 \pm 0,22^{\mathrm{a}}$ & $63,80 \pm 0,22^{\mathrm{b}}(-23,0)$ & $46,20 \pm 0,22^{\mathrm{c}}(-44,3)$ & $46,07 \pm 0,23^{\mathrm{c}}(-44,4)$ & $46,49 \pm 0,24^{\mathrm{c}}(-43,9)$ & $51,89 \pm 0,25^{\mathrm{d}}(-37,4)$ \\
\hline ALH & $1,52 \pm 0,01^{\mathrm{a}}$ & $1,57 \pm 0,01^{\mathrm{b}}(3,3)$ & $1,61 \pm 0,01^{\mathrm{b}}(5,9)$ & $1,42 \pm 0,01^{\mathrm{c}}(-6,6)$ & $1,30 \pm 0,01^{\mathrm{d}}(-14,5)$ & $1,16 \pm 0,01^{\mathrm{e}}(-23,7)$ \\
\hline $\mathrm{BCF}$ & $8,38 \pm 0,20^{\mathrm{a}}$ & $16,93 \pm 0,20^{\mathrm{b}}(102,0)$ & $26,43 \pm 0,20^{\mathrm{c}}(215,4)$ & $21,91 \pm 0,21^{\mathrm{d}}(161,5)$ & $22,96 \pm 0,22^{\mathrm{e}}(174,0)$ & $24,95 \pm 0,23^{\mathrm{f}}(197,7)$ \\
\hline
\end{tabular}

VCL: velocidad curvilínea $\left(\mu \mathrm{m} \mathrm{s}^{-1}\right)$; VSL: velocidad rectilínea $\left(\mu \mathrm{m} \mathrm{s}^{-1}\right)$; VAP: velocidad promedio $\left(\mu \mathrm{m} \mathrm{s}^{-1}\right)$; LIN: índice de linealidad (\%); STR: índice de rectitud (\%); WOB: índice de oscilación (\%); ALH: desplazamiento lateral de la cabeza $(\mu \mathrm{m})$; BCF: frecuencia de entrecruzamiento (Hz). EEM: error estándar de la media. ${ }^{\text {a-f }}$ Diferente superíndice indica diferencias significativas entre frecuencia de fotogramas. $\mathrm{p}<0,05$. / VCL: curvilinear velocity $\left(\mu \mathrm{m} \mathrm{s}^{-1}\right)$; VSL: rectilinear velocity $\left(\mu \mathrm{m} \mathrm{s}^{-1}\right)$; VAP: average path velocity $\left(\mu \mathrm{m} \mathrm{s}^{-1}\right)$; LIN: linearity index (\%); STR: straightness index (\%); WOB: wobble index $(\%)$; ALH: lateral head displacement $(\mu \mathrm{m})$; BCF: beat cross frequency (Hz); SEM: standard error of the mean.

a-f Different superscripts indicate significant differences among frame rates. $\mathrm{p}<0.05$.

\section{Análisis de regresión no lineal}

El modelo de regresión no lineal demostró que la variable más sensible a la frecuencia de fotogramas fue la VCL, esto con un valor alfa de 171,50; seguido de VAP con 72,30. La FR óptima para análisis de cinética estaría sobre $175 \mathrm{fps}$, lo que para efectos prácticos de análisis se consideraría utilizar cámaras de 200 fps. Los demás parámetros de cinética espermática presentaron menor sensibilidad y ALH fue el valor que menor sensibilidad presentó ante el aumento de la FR (alfa = 1,36; Cuadro 2).

\section{Distribución de la cinética espermática según componentes principales}

El conjunto de datos cinéticos se distribuyó en tres vectores de componentes principales (PC) denominados: velocidad-ondulación (PC1), progresividad (PC2) y oscilación (PC3), explicando un 89,39 \% y 94,69 \% de la varianza total para 25 y $200 \mathrm{fps}$, respectivamente. El vector velocidad-ondulación, para ambas FR estuvo representado en mayor medida por la VCL. Otras variables que aportaron información a este componente principal fueron VSL, ALH y VAP. En el caso de 200 fps, la BCF presentó un valor propio de 0,829, indicando que para esta frecuencia de fotogramas hubo un patrón de ondulación más evidente en los conjuntos celulares que a 25 fps. 
Cuadro 2. Nivel asintótico $(\alpha)$, tasa de incremento $(\beta)$, correlación $\alpha / \beta\left(\varrho^{\alpha, \beta}\right)$, y estimación (a 50, 100, 200 y 250 de fotogramas por segundo) de parámetros de cinética espermática de verracos raza Pietrain (Sus scrofa domestica), necesarios para obtener un nivel umbral (95\% de valor máximo), Valencia, España. 2017.

Table 2. Asymptotic level $(\alpha)$, rate of increase $(\beta)$, correlation $\alpha / \beta\left(\varrho^{\alpha, \beta}\right)$, and estimation (at a frame rate of 50, 100, 200, and 250 fps) on Pietrain boar (Sus scrofa domestica) sperm kinematic variables needed to obtain the threshold level (95\% of the maximum), Valencia, Spain, 2017.

\begin{tabular}{|c|c|c|c|c|c|c|c|c|c|}
\hline & \multirow[t]{2}{*}{$\alpha$} & \multirow[t]{2}{*}{$\mathbf{E E}$} & \multirow[t]{2}{*}{$\beta$} & \multirow[t]{2}{*}{$\mathbf{E E}$} & \multirow[t]{2}{*}{$\mathbf{Q}^{\alpha, \beta}$} & \multicolumn{4}{|c|}{$\begin{array}{l}\text { Valores estimados a diferentes fotogramas por } \\
\text { segundo }\end{array}$} \\
\hline & & & & & & 50 & 100 & 200 & 250 \\
\hline VCL & 171,50 & 0,85 & 38,98 & 0,48 & 0,79 & 78,65 & 116,14 & 141,13 & 146,74 \\
\hline VSL & 38,66 & 0,25 & 0,29 & 0,33 & 0,76 & 36,37 & 36,77 & 36,82 & 36,84 \\
\hline VAP & 72,30 & 0,40 & 18,90 & 0,40 & 0,75 & 49,54 & 59,85 & 65,78 & 67,03 \\
\hline LIN & 26,02 & 0,14 & $-28,11$ & 0,17 & 0,88 & 45,65 & 34,47 & 29,95 & 29,11 \\
\hline STR & 58,43 & 0,20 & $-12,30$ & 0,14 & 0,81 & 74,73 & 66,08 & 62,14 & 61,38 \\
\hline WOB & 43,34 & 0,13 & $-16,39$ & 0,11 & 0,82 & 60,15 & 51,06 & 47,04 & 46,28 \\
\hline ALH & 1,36 & 0,01 & $-3,90$ & 0,18 & 0,77 & 1,47 & 1,41 & 1,39 & 1,38 \\
\hline $\mathrm{BCF}$ & 28,41 & 0,18 & 26,44 & 0,53 & 0,76 & 16,74 & 21,81 & 24,89 & 25,56 \\
\hline
\end{tabular}

VCL: velocidad curvilínea $\left(\mu \mathrm{m} \mathrm{s}^{-1}\right)$; VSL: velocidad rectilínea $\left(\mu \mathrm{m} \mathrm{s}^{-1}\right)$; VAP: velocidad promedio $\left(\mu \mathrm{m} \mathrm{s}^{-1}\right)$; LIN: índice de linealidad (\%); STR: índice de rectitud (\%); WOB: índice de oscilación (\%); ALH: desplazamiento lateral de la cabeza ( $\mu$ m); BCF: frecuencia de entrecruzamiento $(\mathrm{Hz})$. EE: error estándar. $\mathrm{p}<0,05$. / VCL: curvilinear velocity $\left(\mu \mathrm{m} \mathrm{s}^{-1}\right)$; VSL: rectilinear velocity $\left(\mu \mathrm{m} \mathrm{s}^{-1}\right)$; VAP: average path velocity $\left(\mu \mathrm{m} \mathrm{s}^{-1}\right)$; LIN: linearity index (\%); STR: straightness index (\%); WOB: wobble index (\%); ALH: lateral head displacement $(\mu \mathrm{m})$; BCF: beat cross frequency $(\mathrm{Hz})$. SE: standard error. $\mathrm{p}<0.05$.

El PC2 denominado progresividad se representó por la LIN y STR. A 25 fps, la variable WOB se asoció con este componente principal $(0,849)$. Para el componente principal 3, llamado vector oscilación, se representó por la BCF $(0,862)$ para 25 fps y por el WOB $(0,985)$ para el caso de 200 fps (Cuadro 3), indicando que a mayor frecuencia de fotogramas, la oscilación de las células se diferenció en mayor medida que a FR menores.

\section{Análisis estructural de subpoblaciones}

Los eyaculados de verraco mostraron un patrón de agrupaciones celulares caracterizadas por la velocidad del movimiento y la progresividad (Figura 2). En el caso de 25 fps, se observó una mayor dispersión de las células respecto de los componentes principales velocidad y progresividad, en el tanto que a $200 \mathrm{fps}$ estos componentes principales condensaron mayoritariamente los espermatozoides. Al incrementar la FR, el aporte de la variabilidad de las variables cinéticas en los componentes principales velocidad y progresividad fue mayor que a $25 \mathrm{~Hz}$ (Figura 2).

Al comparar resultados obtenidos con 25 y 200 fps, el análisis de conglomerados determinó cuatro diferentes subpoblaciones (SP) espermáticas en ambos casos. Las subpoblaciones descritas a $25 \mathrm{~Hz}$, se caracterizaron de la siguiente manera: a) rápida-progresiva (SP1) que mostró una alta velocidad curvilínea, rectilínea y promedio, con índices de linealidad y rectitud altos, sin embargo, el movimiento fue oscilatorio, debido a su elevado WOB; b) lenta-progresiva (SP2), contenía espermatozoides con velocidad baja tal como lo indican sus valores de VCL, VSL y VAP, su movimiento fue progresivo y lineal, debido a sus altos valores de LIN y STR; c) velocidad media no progresiva (SP3), las células presentaron un movimiento medio tal como lo expresa su VCL, pero con movimiento 
Cuadro 3. Vectores propios de componentes principales (PCs) para parámetros de cinética espermática en verracos raza Pietrain (Sus scrofa domestica), a 25 y 200 fotogramas por segundo. Valencia, España. 2017.

Table 3. Eigenvectors of principal components (PCs) for Pietrain boar (Sus scrofa domestica) sperm kinetic parameters in boars at 25 and 200 frames per second. Valencia, Spain. 2017.

\begin{tabular}{|c|c|c|c|c|c|c|}
\hline \multirow[b]{2}{*}{$\begin{array}{l}\text { Componente } \\
\text { principal }^{* / a}\end{array}$} & \multicolumn{3}{|c|}{$25 \mathrm{fps}$} & \multicolumn{3}{|c|}{$200 \mathrm{fps}$} \\
\hline & $\begin{array}{l}\text { Velocidad- } \\
\text { ondulación }\end{array}$ & Progresividad & Oscilación & $\begin{array}{l}\text { Velocidad- } \\
\text { ondulación }\end{array}$ & Progresividad & Oscilación \\
\hline VCL & 0,965 & & & 0,985 & & \\
\hline VSL & 0,798 & & & 0,680 & & \\
\hline VAP & 0,924 & & & 0,836 & & \\
\hline LIN & & 0,974 & & & 0,933 & \\
\hline STR & & 0,801 & & & 0,951 & \\
\hline WOB & & 0,849 & & & & 0,985 \\
\hline ALH & 0,790 & & & 0,959 & & \\
\hline $\mathrm{BCF}$ & & & 0,862 & 0,829 & & \\
\hline Varianza explicada (\%) & 51,34 & 29,04 & 9,01 & 55,08 & 24,13 & 15,47 \\
\hline
\end{tabular}

Varianza total explicada: 89,39\% para 25 fps; 94,68 \% para 200 fps. * Se presentan las variables más importantes en cada PC. Sólo vectores propios $>0,4$ se presentan. ${ }^{a}$ Matriz de componente rotado. VCL: velocidad curvilínea $\left(\mu \mathrm{m} \mathrm{s}^{-1}\right)$; VSL: velocidad rectilínea $(\mu \mathrm{m}$ $\left.\mathrm{s}^{-1}\right)$; VAP: velocidad promedio $\left(\mu \mathrm{m} \mathrm{s}^{-1}\right)$; LIN: índice de linealidad (\%); STR: índice de rectitud (\%); WOB: índice de oscilación (\%); ALH: desplazamiento lateral de la cabeza $(\mu \mathrm{m})$; BCF: frecuencia de entrecruzamiento $(\mathrm{Hz}) /$ Total explained variance: $89.39 \%$ for 25 fps; $94.68 \%$ for 200 fps. * Expresses the more important variables in each PC. Only eigenvectors $>0,4$ are presented. ${ }^{\text {a }}$ Rotated component matrix. VCL: curvilinear velocity $\left(\mu \mathrm{m} \mathrm{s}^{-1}\right)$; VSL: rectilinear velocity $\left(\mu \mathrm{m} \mathrm{s}^{-1}\right)$; VAP: average path velocity $\left(\mu \mathrm{m} \mathrm{s}^{-1}\right)$; LIN: linearity index; (\%); STR: straightness index (\%); WOB: wobble index (\%); ALH: lateral head displacement $(\mu \mathrm{m})$; BCF: beat cross frequency $(\mathrm{Hz})$.
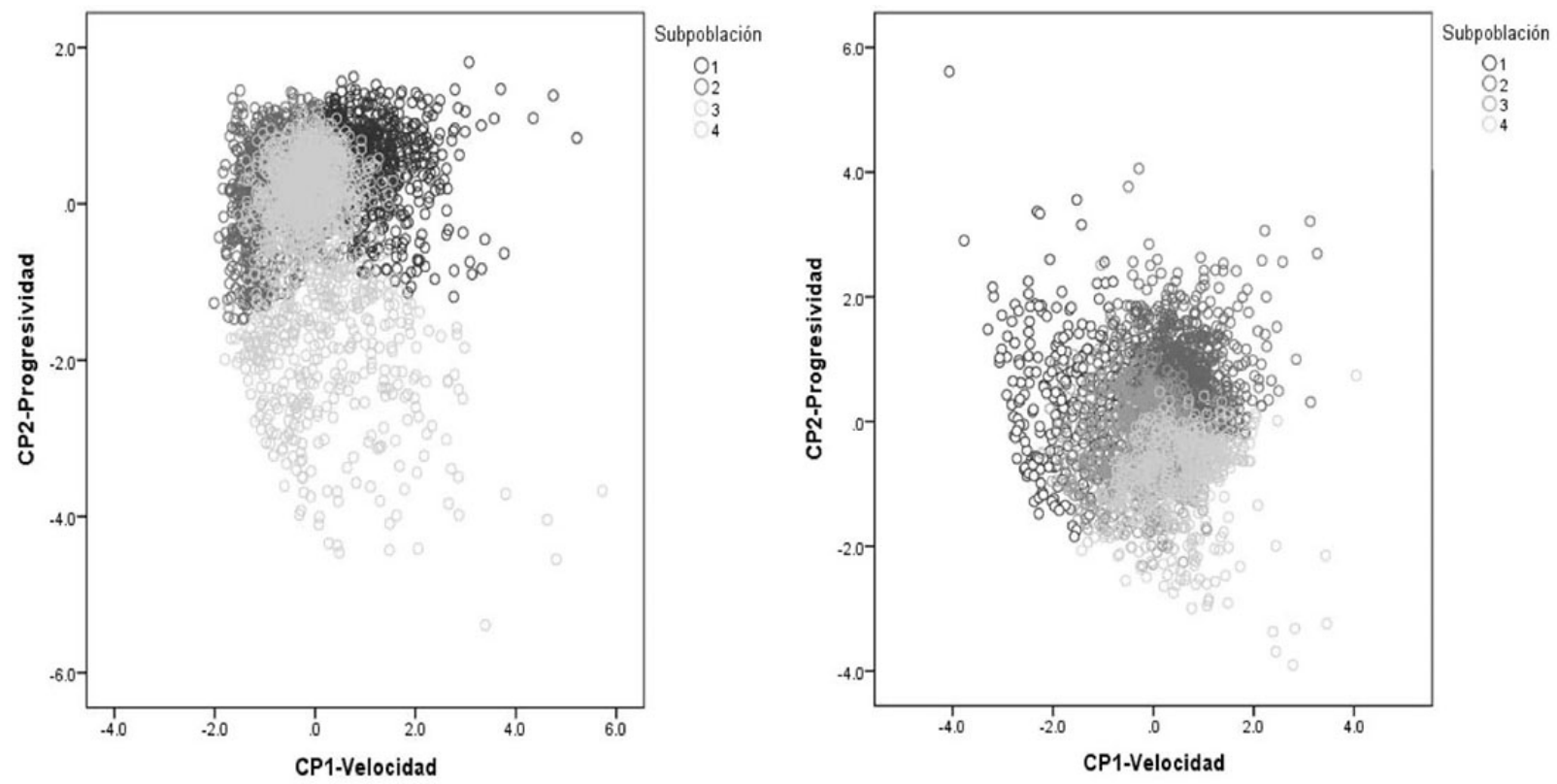

Figura 2. Distribución de las subpoblaciones de espermatozoides en verraco raza Pietrain (Sus scrofa domestica), de acuerdo con los valores de componentes principales a 25 (a) y 200 (b) fps. Valencia, España. 2017.

Figure 2. Distribution of sperm subpopulations in Pietrain boar (Sus scrofa domestica) according to the values of principal components at 25 (a) and 200 (b) fps. Valencia, Spain. 2017. 
ondulatorio no progresivo explicado por su VSL, WOB, BCF y los valores más bajos de LIN y STR; y d) mediaprogresiva (SP4) se caracterizó por describir espermatozoides con velocidad media como lo indica su VCL, VSL y VAP, pero de movimiento circular ondulatorio, debido a sus valores de WOB, BCF y progresividad, por sus altos valores de LIN y STR.

En el caso de las subpoblaciones determinadas con un FR de $200 \mathrm{~Hz}$, la distribución de estas fue diferente tanto porcentualmente como cinéticamente $(\mathrm{p}<0,05)$. La subpoblación 1 se caracterizó por poseer un movimiento rápido, pero no progresivo. La subpoblación 2 presentó un movimiento rápido, progresivo, pero con patrones ondulatorios caracterizados por sus valores altos de BCF. Finalmente, las subpoblaciones 3 y 4 se caracterizaron por presentar velocidades intermedias, sin embargo, la SP3 presentó un patrón de linealidad bajo y movilidad no progresiva, mientras que la SP4 mostró una linealidad alta y el movimiento fue progresivo (Cuadro 4).

Cuadro 4. Subpoblaciones celulares de parámetros de cinética espermática (medias \pm EEM) en verracos Pietrain (Sus scrofa domestica), a 25 y 200 fps. Valencia, España. 2017.

Table 4. Pietrain boar (Sus scrofa domestica) cells subpopulations for kinematic parameters (means \pm SEM) at 25 and 200 fps. Valencia, Spain. 2017.

\begin{tabular}{|c|c|c|c|c|}
\hline \multirow{2}{*}{$\begin{array}{l}\text { Parámetro/ } \\
\text { Subpoblación }\end{array}$} & SP1 & SP2 & SP3 & SP4 \\
\hline & \multicolumn{4}{|c|}{$25 \mathrm{fps}$} \\
\hline VCL & $69,83 \pm 0,54^{\mathrm{a}}$ & $28,93 \pm 0,48^{\mathrm{b}}$ & $49,98 \pm 0,80^{c}$ & $49,45 \pm 0,45^{\mathrm{c}}$ \\
\hline VSL & $62,07 \pm 0,46^{\mathrm{a}}$ & $23,52 \pm 0,41^{\mathrm{b}}$ & $18,76 \pm 0,69^{\mathrm{c}}$ & $40,60 \pm 0,38^{\mathrm{d}}$ \\
\hline VAP & $63,14 \pm 0,47^{\mathrm{a}}$ & $24,78 \pm 0,42^{b}$ & $32,31 \pm 0,70^{c}$ & $40,89 \pm 0,39^{d}$ \\
\hline LIN & $88,85 \pm 0,43^{\mathrm{a}}$ & $79,34 \pm 0,38^{\mathrm{b}}$ & $36,15 \pm 0,64^{\mathrm{c}}$ & $81,50 \pm 0,36^{\mathrm{d}}$ \\
\hline STR & $96,47 \pm 0,35^{\mathrm{a}}$ & $92,58 \pm 0,31^{\mathrm{b}}$ & $58,70 \pm 0,53^{\mathrm{c}}$ & $97,09 \pm 0,29^{\mathrm{a}}$ \\
\hline WOB & $90,44 \pm 0,37^{\mathrm{a}}$ & $84,62 \pm 0,33^{b}$ & $62,65 \pm 0,55^{\mathrm{c}}$ & $82,40 \pm 0,31^{\mathrm{d}}$ \\
\hline ALH & $1,81 \pm 0,02^{\mathrm{a}}$ & $1,12 \pm 0,01^{\mathrm{b}}$ & $2,03 \pm 0,02^{\mathrm{c}}$ & $1,53 \pm 0,01^{\mathrm{d}}$ \\
\hline $\mathrm{BCF}$ & $8,63 \pm 0,09^{\mathrm{a}}$ & $5,53 \pm 0,08^{\mathrm{b}}$ & $7,04 \pm 0,13^{\mathrm{c}}$ & $11,12 \pm 0,07^{\mathrm{d}}$ \\
\hline \multirow[t]{2}{*}{$\mathrm{n}$} & $713(24,02 \%)$ & $902(30,38 \%)$ & $321(10,81 \%)$ & $1033(34,79 \%)$ \\
\hline & \multicolumn{4}{|c|}{$200 \mathrm{fps}$} \\
\hline VCL & $163,93 \pm 2,07^{\mathrm{a}}$ & $186,25 \pm 1,36^{\mathrm{b}}$ & $122,56 \pm 1,39^{c}$ & $101,82 \pm 1,68^{\mathrm{d}}$ \\
\hline VSL & $26,26 \pm 0,80^{\mathrm{a}}$ & $60,47 \pm 0,53^{\mathrm{b}}$ & $25,43 \pm 0,54^{\mathrm{a}}$ & $34,85 \pm 0,65^{\mathrm{c}}$ \\
\hline VAP & $93,66 \pm 1,24^{\mathrm{a}}$ & $89,10 \pm 0,82^{\mathrm{b}}$ & $44,07 \pm 0,83^{c}$ & $54,10 \pm 1,00^{\mathrm{d}}$ \\
\hline LIN & $15,69 \pm 0,38^{\mathrm{a}}$ & $32,56 \pm 0,25^{\mathrm{b}}$ & $20,51 \pm 0,25^{\mathrm{c}}$ & $33,38 \pm 0,30^{\mathrm{b}}$ \\
\hline STR & $27,52 \pm 0,70^{\mathrm{a}}$ & $68,44 \pm 0,46^{\mathrm{b}}$ & $57,62 \pm 0,47^{\mathrm{c}}$ & $63,02 \pm 0,56^{\mathrm{d}}$ \\
\hline WOB & $57,23 \pm 0,41^{\mathrm{a}}$ & $47,68 \pm 0,27^{b}$ & $35,78 \pm 0,27^{\mathrm{c}}$ & $53,27 \pm 0,33^{\mathrm{d}}$ \\
\hline ALH & $1,37 \pm 0,01^{\mathrm{a}}$ & $1,54 \pm 0,01^{\mathrm{b}}$ & $1,19 \pm 0,01^{\mathrm{c}}$ & $1,03 \pm 0,01^{\mathrm{d}}$ \\
\hline $\mathrm{BCF}$ & $25,90 \pm 0,49^{\mathrm{a}}$ & $33,97 \pm 0,32^{\mathrm{b}}$ & $14,65 \pm 0,33^{\mathrm{c}}$ & $16,46 \pm 0,40^{\mathrm{d}}$ \\
\hline $\mathrm{n}$ & $346(14,16 \%)$ & $799(32,71 \%)$ & $769(31,48 \%)$ & $529(21,65 \%)$ \\
\hline
\end{tabular}

Número total de células: 2969 para 25 fps; 2443 para 200 fps. VCL: velocidad curvilínea $\left(\mu \mathrm{m} \mathrm{s}^{-1}\right)$; VSL: velocidad rectilínea $\left(\mu \mathrm{m} \mathrm{s}{ }^{-1}\right)$; VAP: velocidad promedio $\left(\mu \mathrm{m} \mathrm{s}^{-1}\right)$; LIN: índice de linealidad (\%); STR: índice de rectitud (\%); WOB: índice de oscilación (\%); ALH: desplazamiento lateral de la cabeza $(\mu \mathrm{m})$; BCF: frecuencia de entrecruzamiento (Hz). EEM: error estándar de la media. ${ }^{\text {a-d }}$ Dentro de fila, diferentes superíndices indican diferencias significativas entre subpoblaciones espermáticas. p<0,05. / Total number of cells: 2969 for $25 \mathrm{fps} ; 2443$ for $200 \mathrm{fps}$. VCL: curvilinear velocity $\left(\mu \mathrm{m} \mathrm{s}^{-1}\right)$; VSL: rectilinear velocity $\left(\mu \mathrm{m} \mathrm{s}^{-1}\right)$; VAP: average path velocity $(\mu \mathrm{m}$ $\left.\mathrm{s}^{-1}\right)$; LIN: linearity index (\%); STR: straightness index (\%); WOB: wobble index (\%); ALH: lateral head displacement $(\mu \mathrm{m})$; BCF: beat cross frequency $(\mathrm{Hz})$. SEM: standard error of the mean. ${ }^{\mathrm{a}-\mathrm{d}}$ Within row, different superscripts indicate significant differences among sperm subpopulations. $\mathrm{p}<0.05$. 


\section{Discusión}

En el presente estudio, la VCL y VAP presentaron un incremento significativo de sus resultados al incrementar la FR de 25 hasta 250 fps. Esto coincide con los resultados de Valverde et al. (2019a) que presentaron un comportamiento similar (VCL $25 \mathrm{fps}=67,9 \pm 22,6 \mu \mathrm{m} \mathrm{s}^{-1}$; VCL $200 \mathrm{fsp}=187,9 \pm 63,0 \mu \mathrm{m} \mathrm{s}^{-1}$; VAP 25 fps $=$ $64,1 \pm 23,0 \mu \mathrm{m} \mathrm{s}^{-1}$; VAP $200 \mathrm{fps}=98,9 \pm 37,2 \mu \mathrm{m} \mathrm{s}^{-1}$ ) al incrementar de 25 a $200 \mathrm{fps}$. En cuanto a la VSL, Mortimer et al. (1988) indicaron que no existieron diferencias significativas al evaluar con FR entre 25 a 60 fps; sin embargo, Valverde et al. (2019a) mencionaron que existió diferencia entre 25 a 50 fps, pero al incrementar hasta 200 fps no se obtuvo diferencia significativa con respecto a $50 \mathrm{fps}$. Esto podría explicarse por el valor de la tasa de incremento asintótico o valor $\beta$ presentado por la velocidad rectilínea conforme aumentó la frecuencia de fotogramas.

El incremento en los valores de VSL no fue relevante al incrementar la FR según el modelo exponencial de regresión no lineal. En este ensayo se obtuvo como resultado que la VSL no presentó ninguna diferencia significativa al comparar $25 \mathrm{fps}$ con 50, 100, 150, 200 o $250 \mathrm{fps}$. En otras especies se han encontrado resultados que demuestran el mismo comportamiento al incrementar el número de fotogramas por segundo, estas especies son: humano (Morris et al., 1996; Mortimer et al., 1988), perro (Rijsselaere et al., 2004), anguila (Gallego et al., 2013), carpa (Ravinder et al., 1997), toro (Bompart et al., 2019), conejo (Castellini et al., 2011) y ovejo (Castellini et al., 2011).

El índice de linealidad y rectitud presentaron una disminución progresiva al incrementar la FR; no obstante, el WOB presentó el mismo comportamiento hasta $100 \mathrm{fps}$, por encima de este nivel el comportamiento se mantuvo sin variación relevante en sus resultados hasta los 200 fps. En los verracos se ha descrito que los índices LIN y STR disminuyen al incrementar la FR, pero el WOB no se ajusta bien al modelo y sigue un patrón de crecimiento al incrementar el número de fotogramas por segundo (Valverde et al., 2019a).

La amplitud de desplazamiento lateral de la cabeza (ALH) tendió a disminuir conforme aumentó la frecuencia de fotogramas, tal y como lo describieron trabajos previos (Zhu et al., 1994; Kraemer et al., 1998). La frecuencia de entrecruzamiento (BCF) aumentó, debido a que este parámetro es sensible a cambios de dirección por el entrecruzamiento; no obstante, la diferencia fue mayormente significativa con pequeños aumentos de la FR, luego su curva de crecimiento disminuyó (Sellés et al., 2003). La frecuencia de fotogramas es un factor que influye sobre las variables de cinética espermática y debe definirse para estandarizar los análisis CASA entre laboratorios y evitar introducir fuentes de variación externa a los eyaculados.

En este trabajo se obtuvieron cuatros subpoblaciones espermáticas descritas por medio de tres componentes principales, tal como diversos autores lo han definido con similares frecuencias de fotogramas (Flores et al., 2008; Ramió et al., 2008; Valverde et al., 2019a). Sin embargo, la distribución de las subpoblaciones identificadas no fue la misma a 25 que a $200 \mathrm{fps}$, lo cual puede estar relacionado con la variación externa que aportaron factores como la frecuencia de fotogramas en la determinación de las subpoblaciones. A la luz de estos resultados y considerando que para el estudio de la movilidad avanzada de los espermatozoides a mayor FR se puede estimar con mayor precisión la trayectoria real de las células, las subpoblaciones descritas a 200 fps, podrían estar más acordes con las subpoblaciones naturales descritas en el eyaculado.

La idea general de las subpoblaciones de espermatozoides en el eyaculado, radica en el hecho de que hay un grupo de células con patrones de movilidad y cinética que podrían tener mayor probabilidad de llegar al sitio de la fecundación, sin embargo, aún se desconoce el papel real de las demás agrupaciones celulares y su rol en el éxito de aquellas células con mayor probabilidad de fecundar. Algunos trabajos han hecho aportes en relación con las subpoblaciones y la capacidad fecundante de los eyaculados (Holt et al., 1996; Abaigar et al., 1999; Vyt et al., 2008). En cuanto a la fertilidad, diversos autores han demostrado que existe alguna relación entre los parámetros de cinética espermática y la fertilidad (Holt et al., 1997; Broekhuijse et al., 2012; Schulze et al., 2013; Yániz et al., 2017; Tremoen et al., 2018; Winters et al., 2018). 
Es necesario continuar con los trabajos de subpoblaciones espermáticas, minimizando el efecto aquellas fuentes de variación externa que podrían condicionar los resultados de agrupación celular, caracterización de las subpoblaciones celulares naturales y relacionar aquellas subpoblaciones con características de movilidad que puedan tener mayor probabilidad de fecundar el ovocito con los resultados reales de fertilidad en granja.

\section{Conclusiones}

La velocidad del movimiento espermático se vio afectada por la frecuencia de fotogramas. La frecuencia óptima de fotogramas para analizar variables de cinética espermática en verraco con un sistema CASA-Mot fue 200 fps. Una tasa de fotogramas inferior puede provocar una pérdida de la precisión, debido a que se aumenta el intervalo entre imágenes por unidad de tiempo y esto tiende a describir la trayectoria con menor precisión del espermatozoide simulando la trayectoria real.

Los índices de movilidad espermática se vieron reducidos al incrementar el número de fotogramas por segundo. La ALH no sufrió alteraciones relevantes conforme el incremento de la FR, pero la BCF se incrementó sensiblemente con la FR. Si se consideran VCL y VAP como parámetros más sensibles, es recomendable utilizar cámaras de 200 fps para evaluar la cinética espermática de los verracos.

La distribución de subpoblaciones espermáticas varió según la tasa de fotogramas utilizada, por lo que se deben uniformizar criterios de evaluación entre laboratorios respecto de las fuentes de variación externa que podrían condicionar los análisis y resultados posteriores.

Este trabajo puede servir de base para establecer futuras investigaciones con el propósito de estandarizar las evaluaciones y homogenizar los resultados de evaluaciones seminales en verracos entre laboratorios; por ello, futuros ensayos deben basarse en estos resultados.

\section{Conflicto de intereses}

Los autores declaran que no existe conflicto de intereses.

\section{Agradecimientos}

Los autores agradecen al Consejo Nacional para Investigaciones Científicas y Tecnológicas (CONICIT) y al Ministerio de Ciencia, Tecnología y Telecomunicaciones (MICITT), Costa Rica.

\section{Contribución de autores}

Anthony Valverde (AV) estuvo involucrado en todos los experimentos de laboratorio. AV fue el responsable del análisis de datos. Luis Víquez (LV) y Josué Calderón (JC) estuvieron a cargo del análisis de las trayectorias de todas las muestras espermáticas. Vinicio Barquero (VB) y AV participaron en la discusión e interpretación de resultados. VB escribió el borrador inicial de este manuscrito. AV y VB participaron en la redacción del manuscrito final. Todos estuvieron involucrados en la revisión y aprobación de la versión final del manuscrito. 


\section{Referencias}

Abaigar, T., Holt, W., Harrison, R., \& del Barrio, G. (1999). Sperm subpopulations in boar (Sus scrofa) and gazelle (Gazella dama mhorr) semen as revealed by pattern analysis of computer-assisted motility assessments. Biology of Reproduction, 60(1), 32-41. https://doi.org/10.1095/biolreprod60.1.32

Amann, R., \& Hammerstedt, R. (1993). In vitro evaluation of sperm quality: An opinion. Journal of Andrology, 14(6), $397-406$. https://doi.org/10.1002/j.1939-4640.1993.tb03247.x

Amann, R., \& Katz, D. F. (2004). Andrology Lab Corner*: Reflections on CASA After 25 Years. Journal of Andrology, 25(3), 317-325. https://doi.org/10.1002/j.1939-4640.2004.tb02793.x

Amann, R., \& Waberski, D. (2014). Computer-assisted sperm analysis (CASA): Capabilities and potential developments. Theriogenology, 81(1), 5-17. https://doi.org/10.1016/J.THERIOGENOLOGY.2013.09.004

Bompart, D., García-Molina, A., Valverde, A., Caldeira, C., Yániz, J., Núñez de Murga, M., \& Soler, C. (2018). CASAMot technology: how results are affected by the frame rate and counting chamber. Reproduction, Fertility and Development, 30(6), 810-819. https://doi.org/10.1071/RD17551

Bompart, D., Vázquez, R., Gómez, R., Valverde, A., Roldán, E., García-Molina, A., \& Soler, C. (2019). Combined effects of type and depth of counting chamber, and rate of image frame capture, on bull sperm motility and kinematics. Animal Reproduction Science, 209, 106169. https://doi.org/10.1016/J.ANIREPROSCI.2019.106169

Broekhuijse, M. L. W. J., Šoštarić, E., Feitsma, H., \& Gadella, B. M. (2011). Additional value of computer assisted semen analysis (CASA) compared to conventional motility assessments in pig artificial insemination. Theriogenology, 76(8), 1473-1486. https://doi.org/10.1016/j.theriogenology.2011.05.040

Broekhuijse, M. L. W. J., Šoštarić, E., Feitsma, H., \& Gadella, B. M. (2012). Application of computer-assisted semen analysis to explain variations in pig fertility. Journal of Animal Science, 90(3), 779-789. https://doi.org/10.2527/jas.2011-4311

Caldeira, C., Hernández-Ibáñez, S., Valverde, A., Martin, P., Herranz-Jusdado, J. G., Gallego, V., Asturiano, J. F., Dzyuba, B., Pšenička, M., \& Soler, C. (2019). Standardization of sperm motility analysis by using CASA-Mot for Atlantic salmon (Salmo salar), European eel (Anguilla anguilla) and Siberian sturgeon (Acipenser baerii). Aquaculture, 502, $223-231$. https://doi.org/10.1016/j.aquaculture.2018.12.001

Castellini, C., Dal Bosco, A., Ruggeri, S., \& Collodel, G. (2011). What is the best frame rate for evaluation of sperm motility in different species by computer-assisted sperm analysis? Fertility and Sterility, 96(1), 24-27. https://doi.org/10.1016/j. fertnstert.2011.04.096

Fair, S., \& Romero-Aguirregomezcorta, J. (2019). Implications of boar sperm kinematics and rheotaxis for fertility after preservation. Theriogenology, 137, 15-22. https://doi.org/10.1016/j.theriogenology.2019.05.032

Flores, E., Taberner, E., Rivera, M. M., Peña, A., Rigau, T., Miró, J., \& Rodríguez-Gil, J. E. (2008). Effects of freezing/ thawing on motile sperm subpopulations of boar and donkey ejaculates. Theriogenology, 70(6), 936-945. https://doi. org/10.1016/j.theriogenology.2008.05.056

Gallagher, M. T., Cupples, G., Ooi, E. H., Kirkman-Brown, J. C., \& Smith, D. J. (2019). Rapid sperm capture: high-throughput flagellar waveform analysis. Human Reproduction, 34(7), 1173-1185. https://doi.org/10.1093/humrep/dez056 
Gallego, V., Carneiro, P., Mazzeo, I., Vílchez, M., Peñaranda, D., Soler, C., Pérez, L., \& Asturiano, J. (2013). Standardization of European eel (Anguilla anguilla) sperm motility evaluation by CASA software. Theriogenology, 79(7), 1034-1040. https://doi.org/10.1016/J.THERIOGENOLOGY.2013.01.019

Gallego, V., Vílchez, M. C., Peñaranda, D. S., Pérez, L., Herráez, M. P., Asturiano, J. F., \& Martínez-Pastor, F. (2015). Subpopulation pattern of eel spermatozoa is affected by post-activation time, hormonal treatment and the thermal regimen. Reproduction, Fertility and Development, 27(3), 529-543. https://doi.org/10.1071/RD13198

García-Molina, A., Valverde, A., Bompart, D., Caldeira, C., Vendrell, A., \& Soler, C. (2019). Updating semen analysis: a subpopulation approach. Asian Journal of Andrology, 22(1), 118-119. https://doi.org/10.4103/aja.aja_33_19

Hirai, M., Boersma, A., Hoeflich, A., Wolf, E., Foll, J., Aumüller, T. R., \& Braun, J. (2001). Objectively measured sperm motility and sperm head morphometry in boars (Sus scrofa): relation to fertility and seminal plasma growth factors. Journal of Andrology, 22(1), 104-110. http://www.ncbi.nlm.nih.gov/pubmed/11191073

Holt, C., Holt, W., \& Moore, H. (1996). Choice of operating conditions to minimize sperm subpopulation sampling bias in the assessment of boar semen by computer-assisted semen analysis. Journal of Andrology, 17(5), 587-596. https://doi. org/10.1002/j.1939-4640.1996.tb01837.x

Holt, C., Holt, W., Moore, H., Reed, H., \& Curnock, R. (1997). Objectively measured boar sperm motility parameters correlate with the outcomes of on-farm inseminations: results of two fertility trials. Journal of Andrology, 18, 312-323.

Ibanescu, I., Siuda, M., \& Bollwein, H. (2020). Motile sperm subpopulations in bull semen using different clustering approaches - Associations with flow cytometric sperm characteristics and fertility. Animal Reproduction Science, $215,106329$. https://doi.org/10.1016/j.anireprosci.2020.106329

Kaiser, H. F. (1958). The varimax criterion for analytic rotation in factor analysis. Psychometrika, 23, 187-200.

Katz, D. F., \& Dott, H. M. (1975). Methods of measuring swimming speed of spermatozoa. Journal of Reproduction and Fertility, 45(2), 263-272. https://doi.org/10.1530/jrf.0.0450263

Kaufman, L., \& Rousseuw, P. J. (1991). Finding groups in data: An introduction to cluster analysis. Biometrics, $47(2), 788$. https://doi.org/10.2307/2532178

Kime, D., Van Look, K. J., McAllister, B., Huyskens, G., Rurangwa, E., \& Ollevier, F. (2001). Computer-assisted sperm analysis (CASA) as a tool for monitoring sperm quality in fish. Comparative Biochemistry and Physiology Part C: Toxicology \& Pharmacology, 130(4), 425-433. https://doi.org/10.1016/S1532-0456(01)00270-8

Knox, R. V. (2016). Artificial insemination in pigs today. Theriogenology, 85(1), 83-93. https://doi.org/10.1016/j. theriogenology.2015.07.009

Kraemer, M., Fillion, C., Martin-Pont, B., \& Auger, J. (1998). Factors influencing human sperm kinematic measurements by the Celltrak computer-assisted sperm analysis system. Human Reproduction, 13(3), 611-619. http://www.ncbi.nlm. nih.gov/pubmed/9572421

Lopez-Rodriguez, A., Van Soom, A., Arsenakis, I., \& Maes, D. (2017). Boar management and semen handling factors affect the quality of boar extended semen. Porcine Health Management, 3(15), 7981. https://doi.org/10.1186/s40813-017$0062-5$

Lu, J. C., Huang, Y. F., \& Lü, N. Q. (2014). Computer-aided sperm analysis: Past, present and future. Andrologia, 46(4), 329-338. https://doi.org/10.1111/and.12093 
Martínez-Pastor, F., Tizado, E., Garde, J., Anel, L., \& de-Paz, P. (2011). Statistical Series: Opportunities and challenges of sperm motility subpopulation analysis. Theriogenology, 75(5), 783-795. https://doi.org/10.1016/J. THERIOGENOLOGY.2010.11.034

Morris, A. R., Coutts, J. R., \& Robertson, L. (1996). A detailed study of the effect of videoframe rates of 25, 30 and 60 Hertz on human sperm movement characteristics. Human Reproduction, 11(2), 304-310. https://doi.org/10.1093/ HUMREP/11.2.304

Mortimer, S. (2000). CASA - Practical Aspects. Journal of Andrology,21(4), 515-524. https://doi.org/10.1002/J.1939-4640.2000. TB02116.X

Mortimer, D., Serres, C., Mortimer, S., \& Jouannet, P. (1988). Influence of image sampling frequency on the perceived movement characteristics of progressively motile human spermatozoa. Gamete Research, 20(3), 313-327. https://doi. org/10.1002/mrd.1120200307

Mortimer, S., \& Swan, M. (1999). Effect of image sampling frequency on established and smoothing-independent kinematic values of capacitating human spermatozoa. Human Reproduction, 14(4), 997-1004. https://doi.org/10.1093/ humrep/14.4.997

Murtagh, F., \& Legendre, P. (2014). Ward's Hierarchical Agglomerative Clustering Method: Which Algorithms Implement Ward's Criterion? Journal of Classification, 31(3), 274-295. https://doi.org/10.1007/s00357-014-9161-z

Nöthling, J. O., \& dos Santos, I. P. (2012). Which fields under a coverslip should one assess to estimate sperm motility? Theriogenology, 77(8), 1686-1697. https://doi.org/10.1016/j.theriogenology.2011.12.014

Parrilla, I., Perez-Patiño, C., Li, J., Barranco, I., Padilla, L., Rodriguez-Martinez, H., Martinez, E. A., \& Roca, J. (2019). Boar semen proteomics and sperm preservation. Theriogenology, 137, 23-29. https://doi.org/10.1016/j. theriogenology.2019.05.033

Peña, A., Adán, S., Quintela, L., Becerra, J., \& Herradón, P. (2018). Relationship between motile sperm subpopulations identified in frozen-thawed dog semen samples and their ability to bind to the zona pellucida of canine oocytes. Reproduction in Domestic Animals, 53, 14-22. https://doi.org/10.1111/rda.13349

Ramió, L., Rivera, M. M., Ramírez, A., Concha, I. I., Peña, A., Rigau, T., \& Rodríguez-Gil, J. E. (2008). Dynamics of motilesperm subpopulation structure in boar ejaculates subjected to "in vitro" capacitation and further "in vitro" acrosome reaction. Theriogenology, 69(4), 501-512. https://doi.org/10.1016/j.theriogenology.2007.10.021

Ravinder, K., Nasaruddin, K., Majumdar, K. C., \& Shivaji, S. (1997). Computerized analysis of motility, motility patterns and motility parameters of spermatozoa of carp following short-term storage of semen. Journal of Fish Biology, 50(6), 1309-1328. https://doi.org/10.1111/j.1095-8649.1997.tb01655.x

Rijsselaere, T., Van Soom, A., Maes, D., \& de Kruif, A. (2004). Effect of technical settings on canine semen motility parameters measured by the Hamilton-Thorne analyzer. Theriogenology, 60(8), 1553-1568. https://doi.org/10.1016/S0093$691 X(03) 00171-7$

Saravia, F., Núñez-Martínez, I., Morán, J., Soler, C., Muriel, A., Rodríguez-Martínez, H., \& Peña, F. (2007). Differences in boar sperm head shape and dimensions recorded by computer-assisted sperm morphometry are not related to chromatin integrity. Theriogenology, 68(2), 196-203. https://doi.org/10.1016/j.theriogenology.2007.04.052 
Schulze, M., Ruediger, K., Mueller, K., Jung, M., Well, C., \& Reissmann, M. (2013). Development of an in vitro index to characterize fertilizing capacity of boar ejaculates. Animal Reproduction Science, 140(1-2), 70-76. https://doi. org/10.1016/j.anireprosci.2013.05.012

Sellés, E., Gadea, J., Romar, R., Matás, C., \& Ruiz, S. (2003). Analysis of in vitro fertilizing capacity to evaluate the freezing procedures of boar semen and to predict the subsequent fertility. Reproduction in Domestic Animals $=$ Zuchthygiene, 38(1), 66-72. http://www.ncbi.nlm.nih.gov/pubmed/12535333

Soler, C., Contell, J., Bori, L., Sancho, M., García-Molina, A., Valverde, A., \& Segarvall, J. (2017). Sperm kinematic, head morphometric and kinetic-morphometric subpopulations in the blue fox (Alopex lagopus). Asian Journal of Andrology, 19(2), 154-159. https://doi.org/10.4103/1008-682X.188445

Soler, C., Fuentes, M., Sancho, M., García, A., Núñez de Murga, M., \& Núñez de Murga, J. (2012). Effect of counting chamber on seminal parameters, analyzing with the ISASv1@. Revista Internacional de Andrología, 10(4), 132-138. https:// doi.org/10.1016/S1698-031X(12)70069-9

Soler, C., García, A., Contell, J., Segervall, J., \& Sancho, M. (2014). Kinematics and Subpopulations' Structure Definition of Blue Fox (Alopex lagopus) Sperm Motility using the ISAS® V1 CASA System. Reproduction in Domestic Animals, 49(4), 560-567. https://doi.org/10.1111/rda.12310

Spencer, N. H. (2013). Essentials of Multivariate Data Analysis. Chapman and Hall/CRC Press. https://doi.org/10.1201/b16344

Thurston, L., Watson, P., Mileham, A., \& Holt, W. (2001). Morphologically distinct sperm subpopulations defined by Fourier shape descriptors in fresh ejaculates correlate with variation in boar semen quality following cryopreservation. Journal of Andrology, 22(3), 382-394. http://www.ncbi.nlm.nih.gov/pubmed/11330638

Tremoen, N. H., Gaustad, A. H., Andersen-Ranberg, I., van Son, M., Zeremichael, T. T., Frydenlund, K., Grindflek, E., Våge, D. I., \& Myromslien, F. D. (2018). Relationship between sperm motility characteristics and ATP concentrations, and association with fertility in two different pig breeds. Animal Reproduction Science, 193, 226-234. https://doi. org/10.1016/J.ANIREPROSCI.2018.04.075

Valverde, A., Arenán, H., Sancho, M., Contell, J., Yániz, J., Fernández, A., \& Soler, C. (2016). Morphometry and subpopulation structure of Holstein bull spermatozoa: variations in ejaculates and cryopreservation straws. Asian Journal of Andrology, 18(6), 851-857. https://doi.org/10.4103/1008-682X.187579

Valverde, A., Barquero, V., \& Soler, C. (2020). The application of computer-assisted semen analysis (CASA) technology to optimise semen evaluation. A review. Journal of Animal and Feed Sciences, 29(3), 189-198. https://doi.org/10.22358/ jafs/127691/2020

Valverde, A., \& Madrigal-Valverde, M. (2018). Sistemas de análisis computadorizado de semen en la reproducción animal. Agronomía Mesoamericana, 29(2), 469-484. https://doi.org/10.15517/ma.v29i2.30613

Valverde, A., \& Madrigal-Valverde, M. (2019). Evaluación de cámaras de recuento sobre parámetros espermáticos de verracos analizados con un sistema CASA-Mot. Agronomía Mesoamericana, 30(2), 447-458. https://doi.org/10.15517/ am.v30i1.34145

Valverde, A., Madrigal-Valverde, M., Caldeira, C., Bompart, D., Núñez de Murga, J., Arnau, S., \& Soler, C. (2019a). Effect of frame rate capture frequency on sperm kinematic parameters and subpopulation structure definition in boars, analyzed with a CASA-Mot system. Reproduction in Domestic Animals, 54(2), 167-175. https://doi.org/10.1111/rda.13320 
Valverde, A., Madrigal-Valverde, M., Camacho-Calvo, M., Zambrana-Jiménez, A., \& López, L. (2018). Efecto de la composición racial sobre la calidad espermática de verracos. Agronomía Mesoamericana, 29(3), 485. https://doi. org/10.15517/ma.v29i3.32445

Valverde, A., Madrigal-Valverde, M., Castro-Morales, O., Gadea-Rivas, A., Johnston, S., \& Soler, C. (2019b). Kinematic and head morphometric characterisation of spermatozoa from the Brown Caiman (Caiman crocodilus fuscus). Animal Reproduction Science, 207, 9-20. https://doi.org/10.1016/J.ANIREPROSCI.2019.06.011

Valverde, A., Madrigal-Valverde, M., Lotz, J., Bompart, D., \& Soler, C. (2019c). Effect of video capture time on sperm kinematic parameters in breeding boars. Livestock Science, 220, 52-56. https://doi.org/10.1016/j.livsci.2018.12.008

van der Horst, G., Maree, L., \& du Plessis, S. (2018). Current perspectives of CASA applications in diverse mammalian spermatozoa. Reproduction, Fertility and Development, 30(6), 875-888. https://doi.org/10.1071/RD17468

Vásquez, F., Soler, C., Camps, P., Valverde, A., \& García-Molina, A. (2016). Spermiogram and sperm head morphometry assessed by multivariate cluster analysis results during adolescence (12-18 years) and the effect of varicocele. Asian Journal of Andrology, 18(6), 824-830. https://doi.org/10.4103/1008-682X.186873

Verstegen, J., Iguer-Ouada, M., \& Onclin, K. (2002). Computer assisted semen analyzers in andrology research and veterinary practice. Theriogenology, 57(1), 149-179. https://doi.org/10.1016/S0093-691X(01)00664-1

Víquez, L., Barquero, V., Soler, C., Roldan, ERS., \& Valverde, A. (2020). Kinematic sub-populations in bull spermatozoa: A comparison of classical and bayesian approaches. Biology, 9(6), 138. https://doi.org/10.3390/biology9060138

Vyt, P., Maes, D., Quinten, C., Rijsselaere, T., Deley, W., Aarts, M., De Kruif, A., \& Van-Soom, A. (2008). Detailed motility evaluation of boar semen and its predictive value for reproductive performance in sows. Vlaams Diergeneeskundig Tijdschrift, 77(77), 291-298. https://doclib.uhasselt.be/dspace/bitstream/1942/9075/1/sows.pdf

Wilson-Leedy, J. G., \& Ingermann, R. L. (2007). Development of a novel CASA system based on open source software for characterization of zebrafish sperm motility parameters. Theriogenology, 67(3), 661-672. https://doi.org/10.1016/j. theriogenology.2006.10.003

Winters, R. A., Hamilton, D. N., Bhatnagar, A. S., Fitzgerald, R., Bovin, N., \& Miller, D. J. (2018). Porcine sperm binding to oviduct cells and glycans as supplements to traditional laboratory semen analysis. Journal of Animal Science, 96(12), 5265-5275. https://doi.org/10.1093/jas/sky372

Yániz, J., Palacín, I., Caycho, K., Soler, C., Silvestre, M., \& Santolaria, P. (2018). Determining the relationship between bull sperm kinematic subpopulations and fluorescence groups using an integrated sperm quality analysis technique. Reproduction, Fertility and Development, 30(6), 919-923. https://doi.org/10.1071/RD17441

Yániz, J., Soler, C., Alquézar-Baeta, C., \& Santolaria, P. (2017). Toward an integrative and predictive sperm quality analysis in Bos taurus. Animal Reproduction Science, 181, 108-114. https://doi.org/10.1016/j.anireprosci.2017.03.022

Yániz, J., Vicente-Fiel, S., Soler, C., Recreo, P., Carretero, T., Bono, A., Berné, J., \& Santolaria, P. (2016). Comparison of different statistical approaches to evaluate morphometric sperm subpopulations in men. Asian Journal of Andrology, 18(6), 819-823. https://doi.org/10.4103/1008-682X.186872

Zhu, J. J., Pacey, A. A., Barratt, C. L. R., \& Cooke, I. D. (1994). Computer-assisted measurement of hyperactivation in human spermatozoa: Differences between european and american versions of the hamilton-thorn motility analyser. Human Reproduction, 9(3), 456-462. https://doi.org/10.1093/oxfordjournals.humrep.a138527 